\title{
Evaluation of Direct Rapid Immunohistochemical Test (DRIT) of Canis lupus familiaris Hippocampal Touch Impression Smears Using a Monospecific Polyclonal Antibody for Rabies Virus Detection
}

\author{
Mary Jasmin C. Ang, ${ }^{1}$ Daria Llenaresas-Manalo, ${ }^{2}$ Blanca Jarilla, ${ }^{3}$ Laarni Tuason, ${ }^{2}$ Eleonor Avenido, ${ }^{3}$ \\ Catalino Demetria, ${ }^{2}$ Plebeian Medina, ${ }^{2}$ Beatriz Quiambao, ${ }^{4}$ Luz Acosta, ${ }^{3}$ Satoshi Inoue ${ }^{5}$ and Ma. Amelita Estacio ${ }^{1}$ \\ ${ }^{1}$ Department of Basic Veterinary Sciences, College of Veterinary Medicine, University of the Philippines Los Baños \\ ${ }^{2}$ Veterinary Research Department, Research Institute for Tropical Medicine, Philippines \\ ${ }^{3}$ Immunology Department, Research Institute for Tropical Medicine, Philippines \\ ${ }^{4}$ Clinical Research Division, Research Institute for Tropical Medicine, Philippines \\ ${ }^{5}$ National Institute for Infectious Disease, Tokyo, Japan
}

\begin{abstract}
Background. Rabies is an important zoonotic disease that needs to be eradicated worldwide. It is still prevalent in the Philippines, thus development of a relatively affordable but still accurate and rapid post-mortem detection test for the virus is desired, especially in regional laboratories.
\end{abstract}

Methods. The study evaluated the Direct Rapid Immunohistochemical Testing (DRIT) of hippocampal touch impressions of suspected rabid Canis lupus familiaris using monospecific $\mathrm{N}$ protein polyclonal antibody developed by the Research Institute for Tropical Medicine (RITM). One hundred sixty (160) acetone-fixed hippocampal touch impressions were subjected to DRIT.

Results. One hundred thirteen (70.6\%) out of 160 samples tested positive for rabies viral antigen (RVA) and 47 (29.4\%) out of 160 samples tested negative for RVA. No false positive and false negative results were obtained. The results agree with the gold standard, dFAT.

Conclusion. DRIT was able to detect low to high concentrations of RVA in the hippocampal touch impressions based on the grading distribution. DRIT had 100\% sensitivity, specificity and over-all accuracy using monospecific polyclonal antibodies, which suggests its use as a more affordable alternative to the gold standard dFAT.

Key Words: rabies, immunohistochemistry, touch impressions, monospecific polyclonal antibodies, dFAT

Presented at the Philippine Association for Laboratory Animal Science's 26th Annual Scientific Conference, May 2014, Hotel Rembrandt, Quezon City, Philippines.

Corresponding author: Mary Jasmin C. Ang, DVM

Department of Basic Veterinary Sciences

College of Veterinary Medicine

University of the Philippines Los Banos

Los Banos, Laguna Philippines 4031

Telephone: +63 495367512

Email: ang.maryjasmin@gmail.com

\section{Introduction}

Rabies is a zoonotic disease causing more than 59,000 deaths annually worldwide. ${ }^{1}$ As of 2012, the Philippines was ranked $6^{\text {th }}$ in the world with the highest incidence of rabies. Regions in the Philippines with most cases of rabies include region I, VI and IV. ${ }^{2}$ As of 2011, only 28 provinces have attained a rabies-free status. ${ }^{2}$ Eradication of rabies in the country has not been achieved despite years of control and prevention. This is primarily due to difficulty of reporting cases and costly postmortem diagnostic methods. Direct fluorescence antibody testing (dFAT) is currently the gold standard for postmortem rabies diagnosis because of its high specificity $(100 \%)$ and sensitivity $(100 \%){ }^{3}$ dFAT uses touch impressions of the hippocampus and medulla oblongata. It detects the presence of rabies virus antigen (RVA) in the tissue samples as apple green fluorescent inclusion bodies when viewed under the fluorescent microscope. Direct rabies immunohistochemical test (DRIT) is another test employed for postmortem rabies diagnosis and is being developed as an inexpensive alternative test by eliminating the need for specialized equipment. ${ }^{4}$ DRIT also uses touch impressions of the brain for the detection of RVA (magentacolored inclusions) in tissue samples under the light microscope. A previous study of DRIT using monoclonal antibodies as the reagent and the hippocampus, as the tissue sample, found the test to have a specificity of $100 \%$ and a sensitivity of $95 \% .{ }^{5}$ Such results signify the use of DRIT as a more cost effective alternative test for postmortem rabies detection, foregoing the need for fluorescent dye markers and expensive fluorescent microscope. The Research Institute for Tropical Medicine (RITM) recently developed a polyclonal antibody for the rabies virus. This polyclonal antibody developed by RITM is monospecific for the N protein of the rabies virus. A monospecifc polyclonal antibody has higher avidity compared with monoclonal antibodies. Avidity is the over-all binding intensity between antibodies and a multivalent antigen presenting multiple epitopes. ${ }^{6}$ Thus, it is hypothesized that monospecific polyclonal antibodies have 
greater sensitivity than monoclonal antibodies because it offers higher binding affinity with the antigens present in a sample. In order to validate this claim, the recently developed rabies virus polyclonal antibody from RITM was used for direct immunohistochemistry (DRIT) of touch impressions of the hippocampus samples of dogs submitted for rabies virus detection. Results from this study were compared with the results of the previous study which utilized monoclonal antibodies.

\section{Methods}

\section{Hippocampus Samples}

One hundred sixty (160) frozen samples of the hippocampus of suspected rabid dogs submitted to the Research Institute for Tropical Medicine (RITM) for confirmatory rabies diagnosis from January 2011 to December 2012 was used in the study. Samples were already previously tested using dFAT upon submission to RITM. dFAT data were obtained from the records of RITM.

\section{Direct Rapid Immunohistochemistry Test (DRIT)}

\section{Slide Preparation}

Two side-by-side touch impressions of the hippocampus on a single glass slide were made for every sample. For every test run, one negative and one positive control specimen were included to confirm validity of the DRIT method. Twenty (20) samples were prepared for processing every run. Slides were air-dried for $5 \mathrm{~min}$ at room temperature inside a biosafety cabinet.

\section{Fixation}

Following air-drying, slides were immersed in containers with cold acetone for 1 hour for fixation and inactivation of the virus.

\section{Washing}

After fixation of the hippocampal touch impressions, the slides were dip-rinsed 15 times in the wash solution of phosphate buffer solution (PBS) with 1\% Tween 80 (TPBS) to thoroughly wash off any excess fixative.

\section{Reduction of non-specific staining}

The slides were then transferred to containers with 3\% hydrogen peroxide and immersed for $10 \mathrm{~min}$ to block endogenous peroxide to reduce non-specific staining. Afterwards, the slides were dip-rinsed 15 times in TPBS to remove excess hydrogen peroxide.

Incubation in biotinylated anti-rabies polyclonal rabbit antibodies

The slides were placed in a humidity chamber at room temperature. Each slide was incubated with biotinylated anti-rabies $\mathrm{N}$ protein rabbit monospecific polyclonal antibody (developed by RITM) for $10 \mathrm{~min}$. Slides were diprinsed in TPBS 15 times and excess TPBS were blotted off from the edges of the slide and areas surrounding the hippocampal touch impressions.

\section{Incubation in streptavidin-peroxidase complex}

The slides were incubated with streptavidin-peroxidase complex for $10 \mathrm{~min}$ in the humidity chamber at room temperature. Excess reagent was again washed off by diprinsing the slides in TPBS. Blotting off from the edges of the slide and areas surrounding the hippocampal touch impression was done to remove excess TPBS.

\section{Incubation in aminoethylcarbazole (AEC)}

Slides were incubated with aminoethylcarbazole (AEC) in the humidity chamber at room temperature for $10 \mathrm{~min}$. The AEC working solution was prepared just before use to ensure stability of the compound. Excess reagent was removed before dip-rinsing in deionized or distilled water. Aminoethylcarbazole (AEC) is a chromogenic substrate which reacts with the peroxidase reagent to form a colored precipitate. $^{7}$

\section{Counterstaining}

The slides were counterstained with Gill's hematoxylin at a 1:2 dilution with deionized distilled water, for 2 min and immediately dip-rinsed in deionized distilled water. Dip-rinsing in fresh deionized distilled water was done to ensure removal of excess stain. Slides were then transferred to glass containers with fresh distilled water. Slides were processed one by one; slides yet to be processed remained immersed in distilled water to prevent drying of hippocampal impressions.

Mounting

Slides were mounted using a water-soluble mounting medium, 20\% Glycerol-Tris buffered saline $\mathrm{pH}$ 9.0, and cover slipped.

\section{Examination and interpretation}

The slides were examined under a light microscope, using the 20x objective for scanning the field and 40x objective for higher power examination. Rabies virus antigen (RVA) immunoreactivity appeared as red rose or brick red inclusions against the blue neuronal background of the hippocampus. Positive grading of each sample was based on the combined grading of the staining and distribution of RVA.

\section{Staining intensity of rabies virus antigen}

Intensity of RVA red inclusion was graded as follows: +1 intensity $=$ very light but detectable magenta-red inclusion bodies; +2 = slight magenta-red inclusion bodies; +3 intensity = dark magenta-red inclusion bodies; and +4 
intensity $=$ very dark magenta-red inclusion bodies (Niezgoda, 2006). Diminished staining intensity maybe due to non-optimal sample handling leading to denaturation of rabies virus antigens in the tissue sample. ${ }^{8}$ Staining intensity grading system is demonstrated in Figure 2.

\section{Grading distribution of rabies virus antigen}

For each area of the tissue impression smear examined, distribution of the amount of rabies virus antigen present was graded as: $+1=$ small and dust-like magenta-red inclusion bodies present in at least $10 \%$ of the smear, $+2=$ small and dust-like magenta-red inclusion bodies dispersed in each field of the smear (10 to $50 \%$ of the microscopic fields); $+3=$ medium and dust-like magenta-red inclusion bodies dispersed in each field of the smear; and $+4=$ large, medium and dust-like magenta-red inclusion bodies profusely distributed in all fields of the smear. ${ }^{8}$ Distribution of the rabies virus antigen maybe correlated with the stage of clinical infection of the source animal. A higher distribution grade relates to a more advanced clinical phase of the animal. ${ }^{8}$ Distribution grading system is demonstrated in Figure 1.

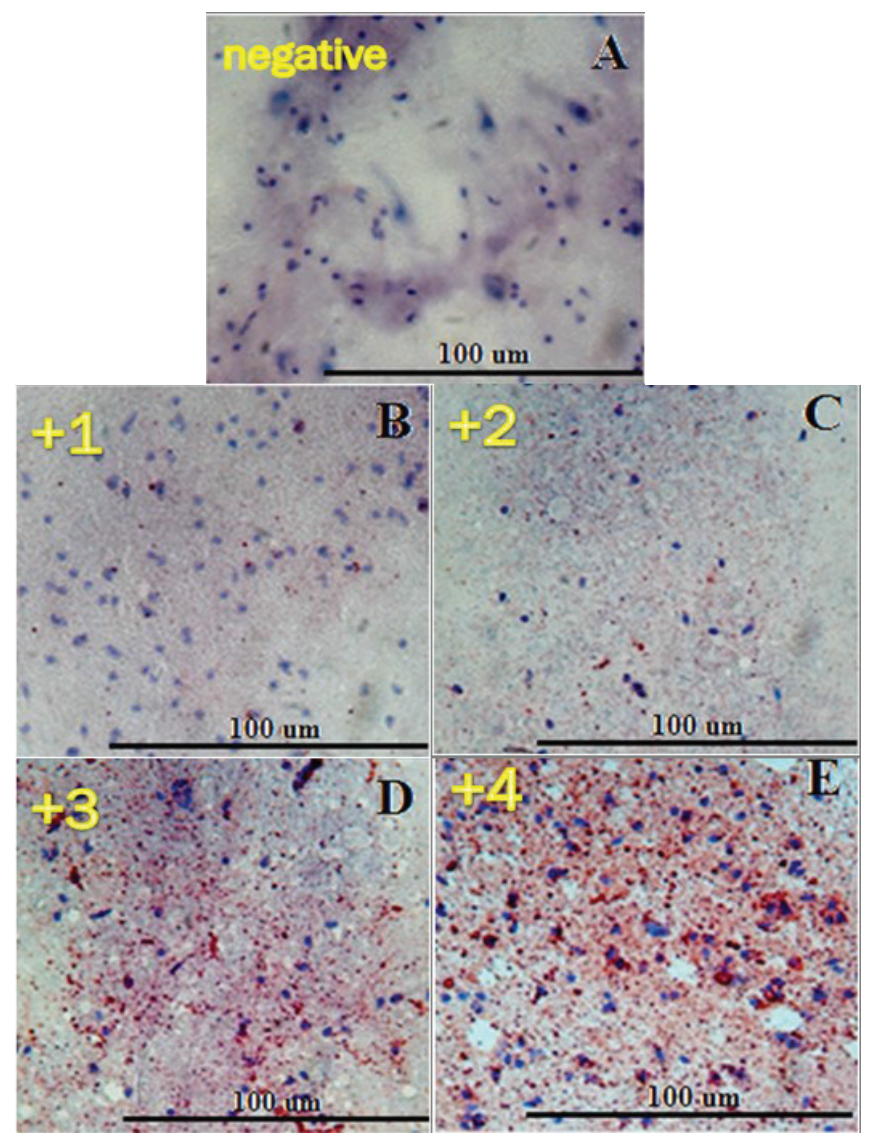

Figure 1. Photomicrograph of acetone-fixed hippocampal touch impressions showing $\mathrm{A}$ : negative; $\mathrm{B}$ : grade $1+; \mathrm{C}$ : grade 2+; D: grade 3+, and E: grade 4+ distribution for rabies virus antigen (red staining inclusions). Also shown are the blue staining neurons.

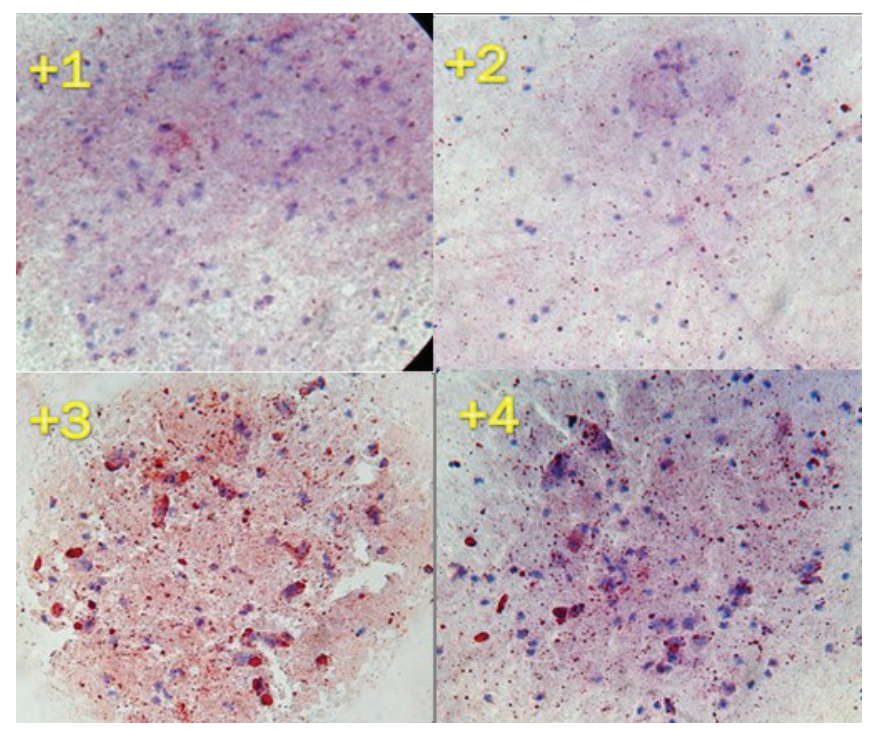

Figure 2. Photomicrograph of acetone-fixed hippocampal touch impressions showing $\mathrm{A}$ : grade 1+; $\mathrm{B}$ : grade 2+; C: grade $3+$ and $D$ : grade $4+$ staining intensity for rabies virus antigen (red staining inclusions). Also shown are the blue staining neurons.

All fields were examined per touch impression smear. A sample is considered positive if at least $10 \%$ of the microscopic fields are positive for inclusion bodies. A positive result obtained from both DRIT and dFAT of a particular sample, renders the sample as positive. A negative result obtained from both DRIT and dFAT of a particular sample, renders the sample as negative. A negative DRIT result with a positive $\mathrm{dFAT}$ result renders the sample as false negative. A positive DRIT result with a negative dFAT result renders the sample as false positive. The resident medical technologist at RITM confirmed all sample readings made by the researcher.

\section{Results and Discussion}

One hundred thirteen (70.6\%) out of 160 samples tested positive for rabies viral antigen (RVA) and 47 (29.4\%) out of 160 samples tested negative for RVA (Table 1). No false positive and false negative results were obtained using DRIT. The present findings show that DRIT of acetone-fixed hippocampal tissue impression smears using the monospecific $\mathrm{N}$ protein polyclonal antibody for the rabies virus was comparably sensitive and specific as that of the gold standard dFAT in detecting the rabies virus.

DRIT of acetone-fixed hippocampal tissue impression smears using the monospecific $\mathrm{N}$ protein polyclonal antibody for the rabies virus was able to accurately detect only true positives and true negatives as confirmed with the gold standard dFAT. Unlike the DRIT study of Caraig (unpublished observations) of hippocampal tissue impression smears using a monoclonal antibody for the rabies virus 
Table 1. Comparison of rabies virus antigen (RVA) detection using DRIT versus dFAT of hippocampal touch impressions of suspected rabid dogs

\begin{tabular}{lll}
\hline & $\begin{array}{c}\text { DRIT Hippocampus } \\
\text { (acetone fixed) }\end{array}$ & $\begin{array}{c}\text { dFAT } \\
\text { Hippocampus }\end{array}$ \\
\hline Positive for RVA & $113(70.6 \%)$ & $113(70.6 \%)$ \\
Negative for RVA & $47(29.4 \%)$ & $47(29.4 \%)$ \\
False positive for RVA & 0 & 0 \\
False negative for RVA & 0 & 0 \\
TOTAL & 160 & 160 \\
\hline
\end{tabular}

Table 2. Predictive value of rabies virus antigen (RVA) detection using DRIT versus the gold standard (dFAT) of hippocampal touch impressions of suspected rabid dogs

\begin{tabular}{llll}
\hline \multirow{2}{*}{ DRIT } & \multicolumn{2}{c}{ DRIT Hippocampus (acetone fixed) } & \multirow{2}{*}{ Predictive Values } \\
\cline { 2 - 3 } & \multicolumn{2}{c}{ Test +} & \multicolumn{2}{c}{ Test - } \\
\hline Test + & 113 & 0 & $113 / 113+0=100 \%$ \\
Test - & 0 & 47 & $47 / 0+47=100 \%$ \\
TOTAL & 113 & 47 & \\
\hline
\end{tabular}

Table 3. Combined staining intensity and distribution grading of RVA positive hippocampal touch impressions of suspected rabid dogs using DRIT and dFAT

\begin{tabular}{cccccc}
\hline & \multicolumn{5}{c}{ Grade of RVA-IR } \\
\cline { 2 - 6 } Method & $\mathbf{+ 1}$ & $\mathbf{+ 2}$ & $\mathbf{+ 3}$ & $\mathbf{+ 4}$ & Total \\
\hline \multirow{2}{*}{ DRIT hippocampus } & 3 & 18 & 40 & 52 & 113 \\
& $(2.7 \%)$ & $(15.9 \%)$ & $(35.4 \%)$ & $(46.0 \%)$ & \\
\multirow{2}{*}{ dFAT hippocampus } & 0 & 3 & 39 & 71 & 113 \\
& & $(2.7 \%)$ & $(34.5 \%)$ & $(62.8 \%)$ & \\
\hline
\end{tabular}

wherein $3 \%$ of the samples were detected as false negative. Another study by Coetzer et al. ${ }^{9}$ using dog brain samples compared monoclonal antibodies versus polyclonal antibodies got the same results, with the DRIT utilising polyclonal antibodies producing $100 \%$ sensitivity and specificity as compared with the 2 types of monoclonal antibodies which had a sensitivity of $83.50 \%$ and $90.78 \%$. This difference may be attributed to the higher avidity of the monospecific $\mathrm{N}$ protein polyclonal antibodies used in the present study. Higher avidity allows the monospecific polyclonal antibodies to elicit higher precipitating action with the antigen and thus demonstrate a higher amount of RVA inclusion bodies in the hippocampal tissue impression smears. ${ }^{9}$

Majority of the samples tested 4+ and 3+ for DRIT while the least number of samples had a $1+$ rating (Table 3 ). dFAT on the other hand was able to detect majority of the samples as $4+$ and $3+$, fewer tissue samples were detected as 2+ compared with DRIT and no brain tissue samples were detected to have a 1+ grading distribution. Differences between the intensity and distribution results between DRIT and DFAT may be attributed to the differences in the viral load of the specific piece of hippocampal mass used in the respective methods. These may be possible because DFAT was done beforehand, upon submission of each sample, while DRIT was done simultaneously per batch for the purpose of this study. This may also be due to the different types of antibodies used between DFAT and DRIT. The high density or distribution of rabies virus antigen in the touch impressions of the hippocampus whether using DRIT or dFAT suggest that majority of the brain samples tested were in the clinical phase of the rabies infection.

The $100 \%$ sensitivity and $100 \%$ specificity of DRIT in detecting true positives and true negatives, respectively were comparably similar with dFAT. Accordingly, both positive and negative predictive values of DRIT were at $100 \%$, using DFAT as the gold standard test as shown in Table 2, indicating that DRIT using polyclonal antibodies is presumably as good as DFAT. The present findings are in agreement with the results of Saturday et al, ${ }^{10}$ Dürr et al. ${ }^{11}$ and Lembo et $\mathrm{al}^{12}$ which also demonstrated that DRIT was comparably sensitive, specific and accurate in detecting the rabies virus antigen in Iraq and Afghanistan; Chad, and Tanzania, respectively. Caraig ${ }^{5}$ on the other hand reported a slightly lower sensitivity of $95 \%$ and over-all accuracy of 96\% with DRIT of hippocampal tissue impression smears using a monoclonal antibody for the rabies virus from CDC.

\section{Conclusion}

These results imply that both DRIT and dFAT can be used to detect low and high concentrations of RVA in hippocampal touch impressions. Moreover, the monospecific $\mathrm{N}$ protein polyclonal antibody for the rabies virus developed by RITM which has a higher binding avidity is highly sensitive, specific and accurate in detecting the rabies virus antigen using DRIT.

\section{Acknowledgement}

The authors thank the Research Institute for Tropical Medicine (RITM) and National Institute for Infectious Disease (NIID), Tokyo, Japan for providing valuable information and materials used in the study.

\section{References}

1. Hampson K, Coudeville L, Lembo T, et al. Estimating the global burden of endemic canine rabies. PLoS Negl Trop Dis. 2015; 9(4).

2. Atienza VC. Animal rabies in the philippines updates . Bureau of Animal Indurstry, Department of Agriculture. 2012:1-7.

3. Robles CG, Miranda NLJ. Comparative evaluation of the rabies fluorescent antibody test and direct microscopic examination at the Research Institute for Tropical Medicine. Phil J Microbiol Infect Dis. 1992; 21(2):69-72

4. Madhusudana SN1, Subha S, Thankappan U, Ashwin YB. Evaluation of a direct rapid immunohistochemical test (dRIT) for rapid diagnosis of rabies in animals and humans. Virol Sin. 2012; 27(5):299-302.

5. Caraig MA. 2012. Direct Rapid Immunohistochemistry Test of Hippocampus and Medulla oblongata Touch Impressions as Diagnostic Assay for Canine Rabies Infection. Unpublished observations. Pp. 23-29 
6. Lipman NS, Jackson LR, Trudel LJ, Weis-Garcia F. Monoclonal versus polyclonal antibodies: distinguishing characteristics, applications, and information resources. ILAR J. 2005; 46(3):258-68.

7. Zarbo RJ, Johnson TL, Kini SR. ABC-immunoperoxidase staining of cytologic preparations: improvement of specificity. Diagn Cytopathol. 1990; 6(2):134-8

8. Niezgoda M, Rupprecht CE. Standard operating procedure for the direct rapid immunohistochemistry test for the detection of rabies virus antigen. National Laboratory Training Network Course. Atlanta: US Department of Health and Human Services, Centers for Disease Control and Prevention; 2006:1-16

9. Coetzer A, Sabeta CT, Markotter W, Rupprecht CE, Nel LH. Comparison of Biotinylated Monoclonal and Polyclonal Antibodies in an Evaluation of a Direct Rapid Immunohistochemical Test for the Routine Diagnosis of Rabies in Southern Africa. PLoS Negl Trop Dis. 2014; 8(9):e3189.

10. Saturday GA, King R, Fuhrmann L. Validation and operational application of a rapid method for rabies antigen detection. US Army Med Dep J. 2009: 42-5.

11. Dürr S, Naïssengar S, Mindekem R, et al. Rabies diagnosis for developing countries. PLoS Negl Trop Dis. 2008; 2(3):e206.

12. Lembo T, Niezgoda M, Velasco-Villa A, Cleaveland S, Ernest E Rupprecht CE. Evaluation of a direct, rapid immunohistochemical test for rabies diagnosis. Emerg Infect Dis. 2006; 12(2):310-3.
The

\section{Natfonal Health Sclence Journal}

lis now indexed in

Sciverse Scopus. 